\title{
Candida: Platelet Interaction and Platelet Activity in vitro
}

\author{
Claudia Eberl $^{\mathrm{a}}$ Cornelia Speth $^{\mathrm{a}}$ Ilse D. Jacobsen ${ }^{\mathrm{b}, \mathrm{c}}$ Martin Hermann ${ }^{\mathrm{d}}$ \\ Magdalena Hagleitner $^{a}$ Hemalata Deshmukh ${ }^{a}$ Christoph G. Ammann ${ }^{e}$ \\ Cornelia Lass-Flörl $^{\text {a }}$ Günter Rambach ${ }^{a}$
}

${ }^{a}$ Division of Hygiene and Medical Microbiology, Medical University of Innsbruck, Innsbruck, Austria; ${ }^{b}$ Research Group Microbial Immunology, Leibniz Institute for Natural Product Research and Infection Biology, Hans Knöll Institute, Jena, Germany; ' Institute for Microbiology, Faculty of Biological Sciences, Friedrich Schiller University Jena, Jena, Germany; ${ }^{d}$ Department of Anesthesiology and Critical Care Medicine, Medical University of Innsbruck,

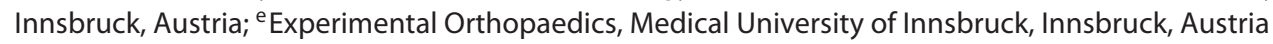

\section{Keywords}

Candida spp · Invasive candidiasis · Platelets · Platelet activation · Antimicrobial peptides $\cdot$ Innate immunity

\section{Abstract}

Over the last 2 decades, platelets have been recognized as versatile players of innate immunity. The interaction of platelets with fungal pathogens and subsequent processes may critically influence the clinical outcome of invasive mycoses. Since the role of platelets in Candida infections is poorly characterized and controversially discussed, we studied interactions of human platelets with yeast cells, (pseudo-)hyphae, biofilms and secretory products of human pathogenic Candida species applying platelet rich plasma and a whole blood model. Incubation of Candida with platelets resulted in moderate mutual interaction with some variation between different species. The rate of platelets binding to Candida (pseudo-) hyphae and candidal biofilm was comparably low as that to the yeast form. Candida-derived secre-
\end{abstract}

\begin{tabular}{ll}
\hline KARGER & $\begin{array}{l}\text { (c) } 2018 \text { The Author(s) } \\
\text { Published by S. Karger AG, Basel }\end{array}$ \\
E-Mail karger@karger.com & This article is licensed under the Creative Commons Attribution- \\
www.karger.com/jin & $\begin{array}{l}\text { NonCommercial-NoDerivatives 4.0 International License (CC BY- } \\
\text { NC-ND) (http://www.karger.com/Services/OpenAccessLicense). } \\
\text { Usage and distribution for commercial purposes as well as any dis- } \\
\text { tribution of modified material requires written permission. }\end{array}$
\end{tabular}

tory products did not affect platelet activity - neither stimulatory nor inhibitory. The small subset of platelets that bound to Candida morphotypes was consequently activated. However, this did not result in reduced growth or viability of the different Candida species. A whole blood model simulating in vivo conditions confirmed platelet activation in the subpopulation of Candida-bound platelets. Thus, the inability of platelets to efficiently react on Candida presence might favor fungal survival in the blood and contribute to high morbidity of Candida sepsis.

(C) 2018 The Author(s)

Published by S. Karger AG, Basel

\section{Introduction}

Candidaemia is still a serious condition with high incidence particularly in intensive care units and is associated with high morbidity and mortality, prolonged hospital stay and considerable healthcare costs $[1,2]$. Recent studies showed an increasing burden of invasive candidiasis 
(IC) in some centers in US and Europe especially observed in the elderly, and this might also be related to debilitated or immunosuppressed patients surviving formerly fatal diseases [1]. Although fluconazole is still active against most of the Candida species, the ongoing trend to rising resistance led to the recommendation of echinocandins as first-line treatment $[3,4]$. Candida (C.) albicans still accounts for approximately half of the IC cases, but in most parts of the world, a progressing shift to non-albicans species such as C. glabrata, C. parapsilosis, C. krusei and C. tropicalis is observed, probably resulting from the increased exposure to azoles [1,3]. Moreover, C. auris has emerged as a new threat in recent years, a species with multiple intrinsic resistances against common antifungals [5]. Despite improved therapeutical treatment and new antimycotic drugs, the mortality rate of IC remains high $(\geq 30 \%)$ [1], which highlights the urgent need for a better understanding of interactions between Candida spp. and the various elements of the immune system.

Platelets are primarily known for their crucial role in hemostatic processes but are also versatile players of the immune network, participate in inflammatory processes and can support the defense against infections. As a part of innate immunity, they express receptors for foreign structures, undergo activation and subsequently release antimicrobial peptides and a variety of bioactive mediators including cytokines and chemokines, which interact with other elements of the innate and acquired immune network [6-9].

There are only few data available on the interactions of platelets and fungi $[10,11]$. Relevance of platelets for antifungal defenses can be deduced by the fact that thrombocytopenia represents a highly significant risk factor for fungal infections [12-14]. Decreased numbers of platelets are frequently found in candidaemia patients, but it remains unclear whether this may result from interactions of platelets with the fungus, as thrombocytopenia is also described as an adverse effect of antimycotics or was present ahead of infection. However, in former studies, we observed that platelets that are exposed to Aspergilli or secreted fungal factors undergo activation [15-17]. Aspergilli exposed to platelets showed delayed germination, impaired hyphal elongation, and downregulated genes of cell wall synthesis $[15,16,18]$.

For Candida albicans, it is known that this species interacts with platelets in vitro $[19,20]$, and high numbers of microparticles shed from activated platelets are detected in patients with severe Candida sepsis [21]. Candida albicans strains that are susceptible in vitro to plateletderived microbicidal peptides (PMP) show decreased vir- ulence in a rabbit model [22]. In vitro data imply that PMP enhance the fungicidal effect of fluconazole, a firstline candidastatic agent [23]. The mycotoxin gliotoxin, which is produced by Aspergillus and Candida species, inhibits platelet functionality and thus might contribute to yeast survival in the bloodstream during sepsis [24]. However, most of these analyses were done with laboratory strains; moreover, the studies were restricted to the species C. albicans and concentrated on the yeast form.

For these reasons, we tested clinical Candida isolates from different species in vitro and evaluated their capacity to stimulate platelets. Our results show that only a minor part of the platelets bound to Candida yeast cells and underwent activation, with some minor variation between the species. Similar results were obtained with hyphae and biofilms, whereas platelets did not react to Candida-derived secretory products. In accordance with the lack of efficient platelet activation, no platelet-derived antifungal effect on Candida could be observed. Taken together, the limited direct reaction of platelets to different Candida morphotypes and secretory products might favor fungal survival in the blood and crucially contribute to lethal outcome of disseminated Candida infections.

\section{Materials and Methods}

\section{Antibodies and Chemicals}

All antibodies were purchased from BioLegend, thrombin and RPMI medium from Sigma-Aldrich and Concanavalin A from LifeTechnologies/Thermo Fisher Scientific. Calcofluor white (Fungi-FluorTM Kit) was purchased from Polysciences Inc. and skim milk from Becton Dickinson.

\section{Fungal Isolates, Cultivation and Staining}

Different Candida strains, isolated from clinical specimens of candidaemia patients with various underlying diseases (mainly leukemia), were used (Table 1). For comparison, two C. albicans reference strains (SC5314 and SN152) $[25,26]$ as well as a clinical isolate of Aspergillus fumigatus were used in some experiments.

Fungi were grown on Sabouraud Dextrose Agar (BD Diagnostic Systems). For the experiments with yeast cells, Candida colonies from the plates were resuspended in RPMI medium and added to the experimental assays. To induce hyphae formation, yeast cells of the different Candida species were cultivated overnight in $\mathrm{RPMI}$ at $37^{\circ} \mathrm{C}$. For microscopy, hyphae were attached to polylysine-coated coverslips, unbound fungi were removed by washing with PBS. For biofilm formation, Candida yeast cells were incubated for 2 days on polylysine-coated cover slips.

Calcofluor stain of fungi was used for flow cytometry, microscopic analysis and fungal biomass quantification. The staining solution was added in a dilution of 1:10 (v/v) to the fungus and incubated for $3 \mathrm{~min}$, followed by washing with PBS. Biofilms were additionally stained with $200 \mu \mathrm{g} / \mathrm{ml}$ fluorescein-conjugated Concanavalin A for 30 min and subsequently washed with PBS. 
Table 1. Fungal isolates used in the study

\begin{tabular}{lll}
\hline Species & Source & $\begin{array}{l}\text { Internal } \\
\text { nomenclature }\end{array}$ \\
\hline Candida albicans & Reference strain & SC5314 \\
Candida albicans & Reference strain & SN152 \\
Candida albicans & Blood culture & CA1 \\
Candida albicans & Tissue biopsy & CA3 \\
Candida auris & Blood culture & CAU1 \\
Candida glabrata & Blood culture & CG1 \\
Candida krusei & Blood culture & CK1 \\
Candida lusitaniae & Blood culture & CL1 \\
Candida parapsilosis & CSF & CP1 \\
Candida rugosa & Blood culture & CR1 \\
Candida tropicalis & Blood culture & CT1 \\
Aspergillus fumigatus & Lung biopsy & A22 \\
\hline
\end{tabular}

\section{Preparation of Fungal Supernatants and Quantification}

of Fungal Growth

To obtain fungal supernatants (SN), $10^{4}$ Candida cells or conidia of $A$. fumigatus were inoculated in RPMI medium and incubated at $37^{\circ} \mathrm{C}$. After 2 days, fungal cells were removed by centrifugation and filtration through Spin-X filters (Corning Life Sciences). The fungal supernatant was frozen at $-20^{\circ} \mathrm{C}$ for further use.

To analyze the platelet effect on fungal growth, Candida yeast cells were incubated for $18 \mathrm{~h}$ with platelets or platelet lysate in varying concentrations. The respective volume of platelet-poor plasma (PPP) was added to control samples. Quantification of fungal biomass was performed by calcofluor staining and subsequent measurement with a fluorescence plate reader (TECAN Infinite M200 Pro). For killing assays, $5 \mu \mathrm{g} / \mathrm{mL}$ propidium iodide were added to each well; fluorescence was determined with the fluorescence plate reader.

\section{Platelet Isolation and Platelet Lysates}

All studies were approved by the local Ethics Committee. Blood was obtained with informed consent from healthy volunteers. Human platelets were prepared as concentrates by thrombocytapheresis with Amicus cell separator (Baxter) by the Department of Immunology and Blood Transfusion (Medical University of Innsbruck). Alternatively, venous blood was withdrawn with a trisodium citrate blood collection system (Sarstedt). For whole blood as platelet source, it was used within $1 \mathrm{~h}$ after collection to prevent loss of platelet function. Platelet-rich plasma (PRP) was prepared by centrifugation at $135 \mathrm{~g}$ for $15 \mathrm{~min}$ at room temperature. To obtain PPP, the concentrate or PRP was centrifuged at $1,500 \mathrm{~g}$ for $15 \mathrm{~min}$ to remove platelets.

To obtain platelet lysate, platelet concentrate was frozen at $-20^{\circ} \mathrm{C}$ and immediately thawed at $37^{\circ} \mathrm{C}$ for 3 consecutive cycles; platelet membranes were removed by centrifugation at $4,000 \mathrm{~g}$ for $30 \mathrm{~min}$.

\section{Platelet Interaction, Activity and Viability}

To quantify platelet interaction with Candida yeast cells, PRP was stained with a PE-conjugated CD41-antibody, and a fivefold number (in relation to platelets) of calcofluor-labelled yeast cells was added. After $60 \mathrm{~min}$, sample was fixed with $1 \%$ formalin, and
CD41-positive platelets also positive for calcofluor were quantified by flow cytometry (FACSCanto flow cytometer, BD Diagnostics).

To monitor platelet activation, PRP was incubated in RPMI with thrombin $(0.1 \mathrm{U})$, calcofluor-labelled Candida yeast cells, or Candida supernatant, respectively, for $90 \mathrm{~min}$. Platelet activity was examined by detection of platelet activation markers CD62P and CD63 using conjugated antibodies by flow cytometry.

To assess a potential influence of other blood cells, platelet binding and activation experiments were alternatively performed using whole blood (trisodium citrate, see above) instead of PRP. The numbers of platelets corresponded to those of the experiments with PRP, and also the experimental procedures using whole blood complied with those for PRP (see above).

To measure platelet viability, an amine-reactive dye (LIVE/ DEAD $^{\circledR}$ Fixable Violet Dead Cell stain kit; Thermo Fisher Scientific) was used according to manufacturer's instructions. The dye was added to the platelets previously labelled with fluorescent $\alpha$-CD41 antibody, and incubated for $30 \mathrm{~min}$. The samples were washed with PBS, fixed with $1 \%$ formalin and analyzed by flow cytometry.

\section{Fluorescence Microscopy, Confocal and Scanning}

\section{Electron Microscopy}

For fluorescence microscopy, human platelets stained with PElabelled $\alpha$-CD41 antibody were added to Candida yeast cells, (pseudo-)hyphae or a biofilm. After $90 \mathrm{~min}$, the samples were washed and embedded in mounting medium containing 4', 6-Diamidino2-phenylindole dihydrochloride (DAPI; Sigma-Aldrich). For comparison, platelets were incubated with Aspergillus fumigatus conidia.

For electron microscopy, fungal conidia or hyphae were incubated with platelets for $90 \mathrm{~min}$. The samples were washed, fixed in $2.5 \%$ glutaraldehyde, gradually dehydrated with ethanol, followed by critical point drying (CPD 030, Bal-Tec), sputter-coating with $10 \mathrm{~nm} \mathrm{Au} / \mathrm{Pd}(\mathrm{Bal}-\mathrm{Tec})$, and examination with a field-emission scanning electron microscope (Gemini 982; Zeiss).

Confocal microscopy was performed with a spinning disc confocal microscopic system (Ultra VIEW VoX; Perkin Elmer) connected to a Zeiss AxioObserver Z1 inverted microscope (Zeiss).

\section{Statistical Analysis}

All experiments were performed in triplicates and repeated at least 3 times with different platelet donors. Results presented are the mean \pm SE from one representative experiment. Statistical analyses were performed using one-way analysis of variance (Graph-prism software). Values of $p<0.05$ were considered statistically significant.

\section{Results}

\section{Interaction of Human Platelets with Candida}

\section{Yeast Cells}

Quantification of human platelets binding to C. albicans by flow cytometry showed that only $0.3-1 \%$ of the platelets adhered to fungal cells of the different isolates, although a fivefold excess of the yeast cell had been added (Fig. 1a). No significant difference was observed between the clinical isolates (CA1, CA3) and the reference strains 
Fig. 1. Interactions of platelets with Candida yeast cells. a Platelets were stained with an FITC-labelled a-CD41 antibody, followed by the incubation for $90 \mathrm{~min}$ with a fivefold excess of calcofluor-stained yeast cells of clinical isolates of C. albicans (CA1, CA3), C. glabrata (CG1), C. auris (CAU1), C. krusei (CK1), C. lusitaniae (CL1), C. parapsilosis (CP1), C. rugosa (CR1) or C. tropicalis (CT1). C. albicans isolates SC5314 and SN152 were used as reference strains. Percentage of platelets bound to the different Candida species was evaluated by flow cytometry. b Candida-platelet interactions were visualized by confocal microscopy; representative results are shown for Candida albicans (CA1, CA3), tropicalis (CT1), and parapsilosis (CP1). Calcofluor staining was used for the fungi (blue), platelets were marked with an FITC-labelled $\alpha$-CD41 antibody (green). Binding experiments were performed in triplicates and repeated at least 3 times with different platelet donors; representative results are shown here.

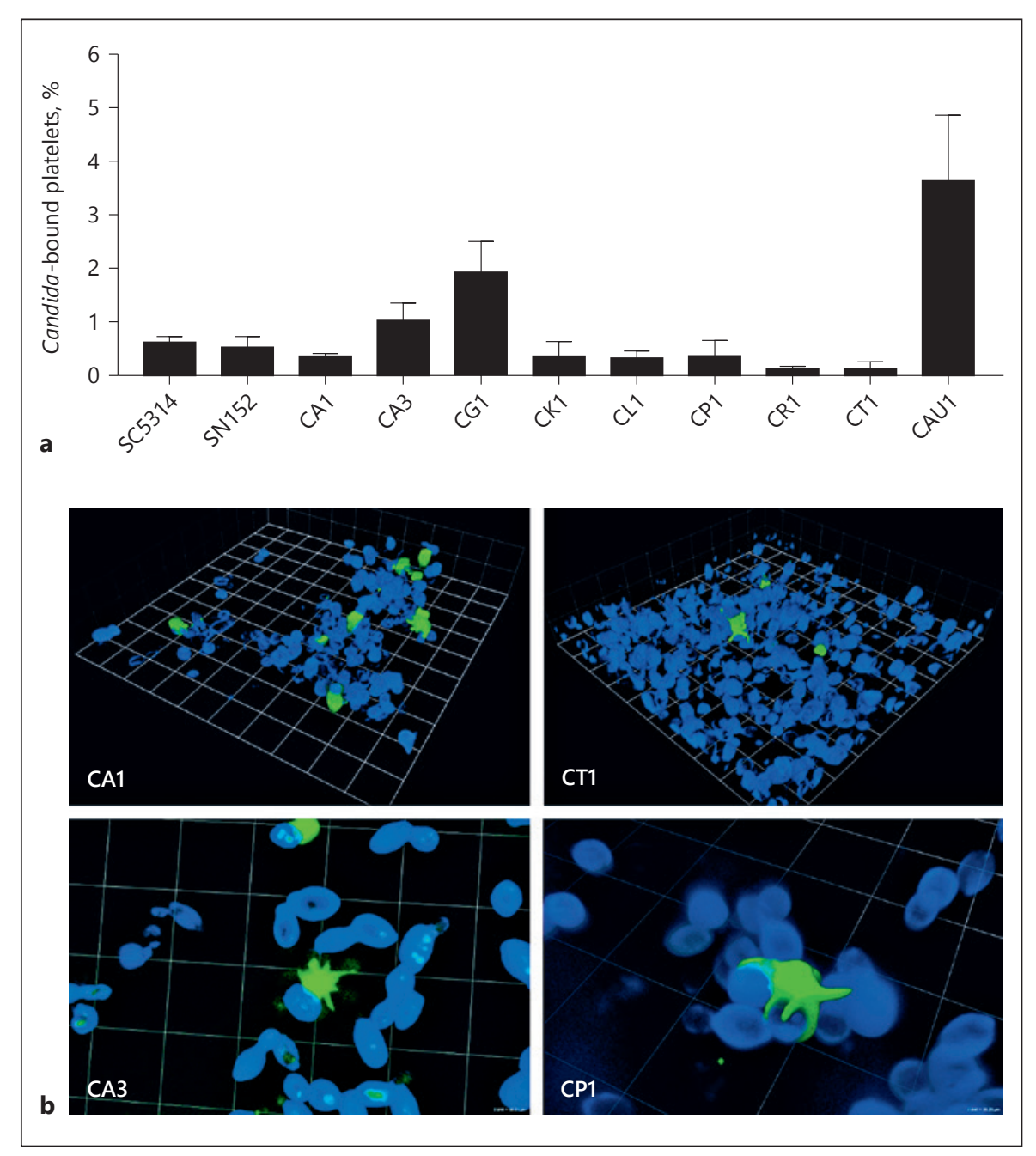

(SC5314, SN152). A comparison with other pathogenic Candida species revealed low variance; only C. glabrata and $C$. auris displayed a higher platelet interaction ( 2 and $3.8 \%$, respectively) rate, whereas less than $0.1 \%$ of platelets bound to C. rugosa and C. tropicalis (Fig. 1a). The precise percentages varied between different donors, but the tendency was similar. Confocal microscopy confirmed the limited physical interactions between platelets and Candida as shown for C. albicans and C. tropicalis as examples (Fig. 1b, upper row). More detailed viewing visualized that the few bound platelets undergo a firm attachment to the fungal surface with their pseudopodia (Fig. 1b, lower row).

\section{Platelet Activation by Candida Yeast Cells as a \\ Function of Interaction}

Incubation with Candida yeast cells of all tested fungal species did not induce general platelet activation, as shown by flow cytometry quantification of CD62P, a marker for release of alpha granules (Fig. 2a). In the small subpopulation of Candida-bound platelets, however, the direct contact to the Candida surface did induce an increase of the activation marker CD62P. Interestingly, there was a tendency towards an inverse correlation between the speciesspecific rate of platelet binding and activation; species with low binding affinities, such as $C$. tropicalis, $C$. rugosa and C. krusei demonstrated rather high platelet activation capacity, whereas platelets adhered to C. glabrata or $C$. auris exhibited only low stimulation levels (Fig. 2a).

Activation of the subset of yeast-bound platelets was also confirmed by confocal microscopy analysis. Both appearance of pseudopodia formation, which is characteristic for activated platelets, and increase of CD62P signal could be demonstrated (Fig. 1b, 2b). Similar results were achieved with CD63, a platelet marker for the activation-induced release of the second platelet granule type, the dense granules: While no in- 

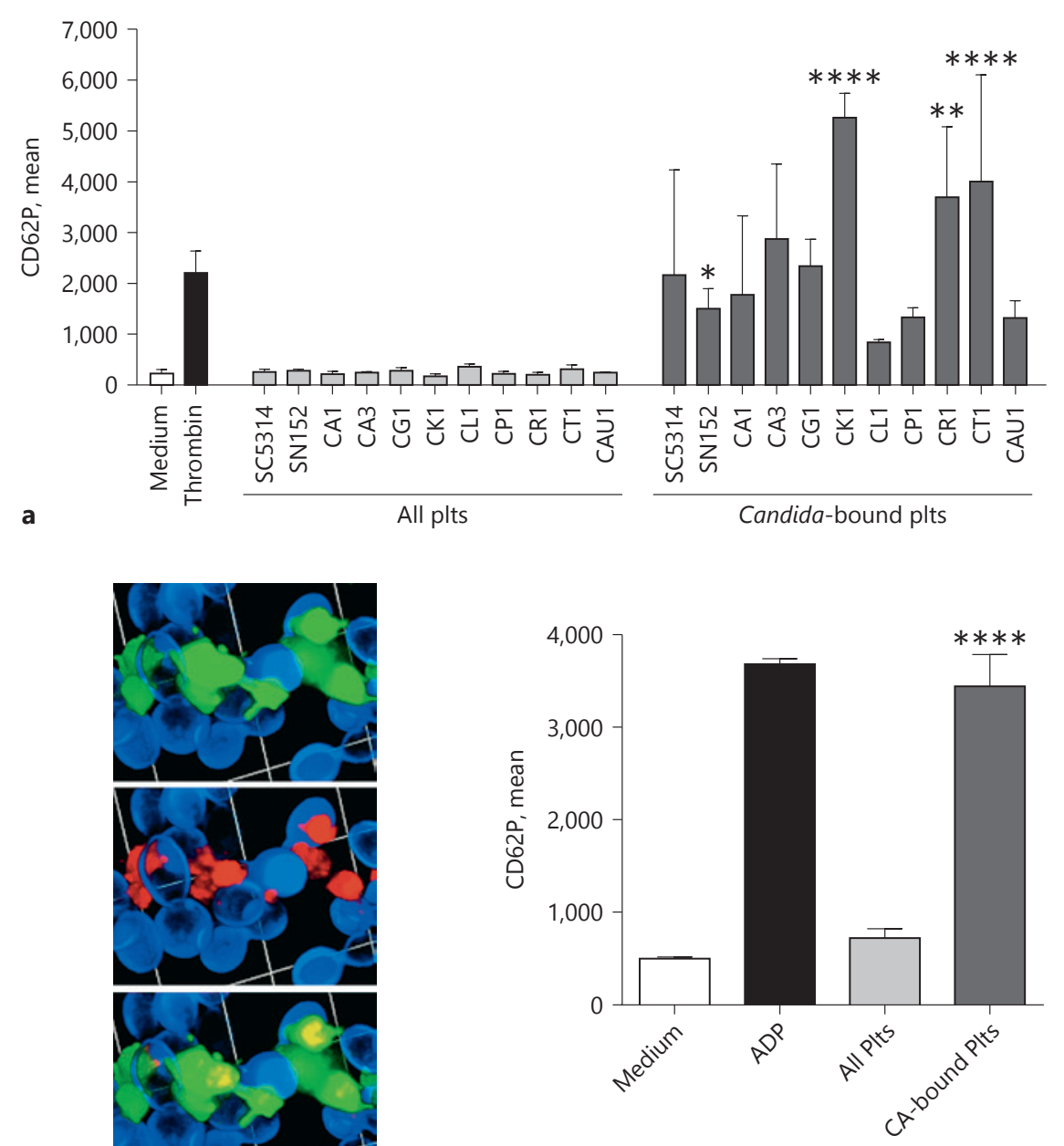

b

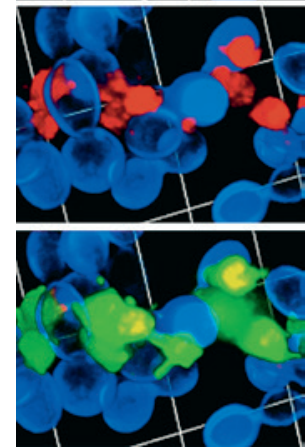

Fig. 2. Platelet activation by Candida yeast cells. a Platelets (plts) were stained with an FITC-labelled $\alpha$-CD41 antibody, followed by the incubation for $90 \mathrm{~min}$ with medium, thrombin, or a 5 -fold excess of calcofluor-stained yeast cells of C. albicans (CA1, CA3), C. glabrata (CG1), C. auris (CAU1), C. krusei (CK1), C. lusitaniae (CL1), C. parapsilosis (CP1), C. rugosa (CR1) or C. tropicalis (CT1). C. albicans isolates SC5314 and SN152 were used as reference strains. Exposure of the platelet activation marker CD62P was assessed by flow cytometry and expressed as mean fluorescence intensity (mean). b Platelet activation was confirmed by confocal microscopy using PE-labelled $\alpha$-CD62P antibody for activated platelets (red); platelet presence is shown in upper image (green), activity in the

crease could be measured when analysing the whole platelet population, the CD63 mean was significantly higher in the subset of yeast-bound platelets (data not shown).

To evaluate the influence of other blood-derived cellular and soluble immune elements on Candida- middle, and overlay in bottom image. $\mathbf{c}$ Whole blood was incubated in RPMI medium alone or additionally with ADP as a positive control, or calcofluor-labelled C. albicans (CA1) yeast cells (fivefold excess over platelet number), respectively, for $90 \mathrm{~min}$. Activity was evaluated by flow cytometry analysis of CD62P. For both PRP and whole blood, activation of all platelets (CD41+ events) was compared with the subpopulation of Candida-bound platelets (calcofluor+ and CD41+ events). All experiments were performed in triplicates and repeated at least 3 times with different platelet donors; representative results are shown here. Activity of samples with Candida were compared to medium control by one-way analysis of vari-

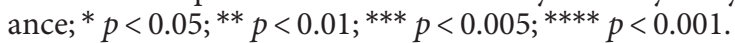

platelet interaction, further experiments were performed using whole blood instead of PRP. The percentage of platelets binding to C. albicans in whole blood was comparable to PRP of the same donor (data not shown). Similar to PRP, C. albicans induced no or only marginal overall platelet activation in whole-blood, but 

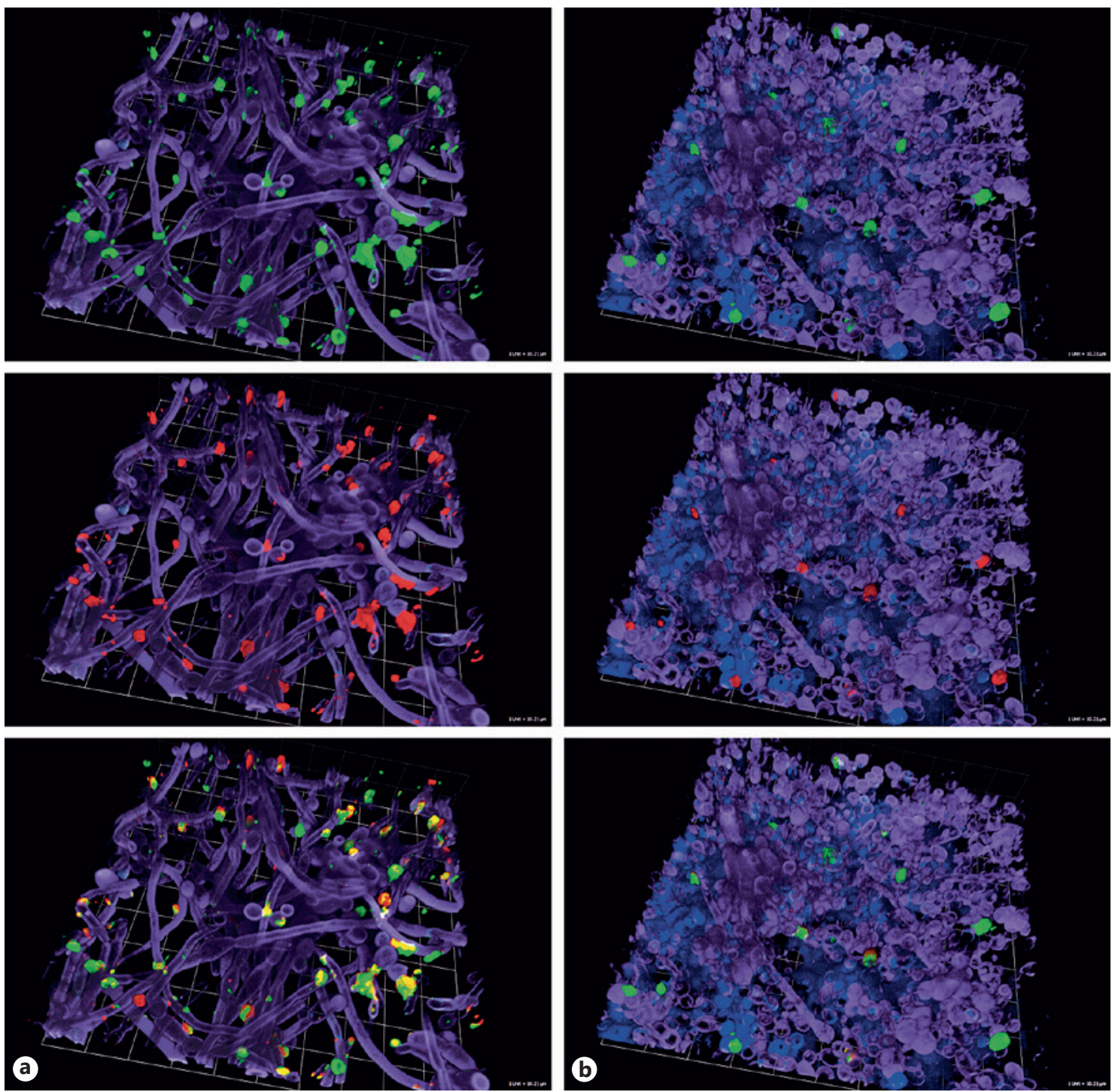

Fig. 3. Activity of platelets incubated with Candida (pseudo-)hyphae and biofilms. Confocal microscopy analysis of binding and activity of platelets incubated with Candida albicans (pseudo-)hyphae (a) and biofilm (b) was performed using calcofluor staining for the fungi (purple), FITC-labelled a-CD41 antibody staining for all platelets (green, upper row), PE-labelled $\alpha$-CD62P antibody for activated platelets (red, middle row) and concanavalin A for biofilm polysaccharides (blue). Images in the bottom row show an overlay of all colours. was able to significantly activate the platelet subpopulation that physically interacted with the yeast cells (Fig. 2c).

Platelet Activation by Different Candida Morphotypes

We also evaluated for selected Candida species whether platelet adhesion and activation was more pronounced after contact with Candida pseudohyphae or hyphae of selected Candida species. Since no flow cytometry analysis is possible with these fungal structures, we focused on

Candida and Platelet Interactions confocal microscopy. Figure 3 a shows that only few platelets attached to C. albicans filaments. Again, those platelets that interacted with the hyphal structures were stimulated, as shown by strong presence of the activation marker CD62P on the platelet surface. A similar result could be achieved by the incubation of C. albicans biofilms with platelets (Fig. 3b).

Interestingly, no clear difference was visible for binding to (pseudo-)hyphae from different Candida species. Comparable amounts of platelets were found to adhere to 
C. albicans and C. tropicalis and, similarly, only minor platelet binding was visible using biofilms of $C$. albicans and C. glabrata (data not shown).

\section{Platelets Are Not Affected by Candida-Derived Soluble Factors}

Since Aspergillus fumigatus was described to secrete compounds that activate platelets [17], we evaluated if a soluble Candida-secreted compound is capable to stimulate platelets. Incubation of human platelets with increasing concentrations of $C$. albicans culture supernatant did not result in enhanced exposure of the activation marker CD62P (Fig. 4a, left). Similarly, the supernatants of the C. albicans reference isolate SC5314 or of other non-albicans Candida species did not activate the platelets (Fig. 4a, right). To further investigate the striking difference between the secretory products of A. fumigatus and Candida, we tested whether supernatants affected platelet viability. While the A. fumigatus supernatant did not only activate but also considerably damaged the platelets, even the highest concentrations of $C$. albicans supernatant did not induce any cellular damage (Fig. 4b, left); this result could be confirmed by testing the other C. albicans and non-albicans supernatants (Fig. 4b, right). Furthermore, Candida spp. supernatants showed no inhibitory effect on platelets, as their ability to be activated by thrombin remained unchanged in presence of the Candida-derived soluble factors (Fig. 4c).

\section{Platelet Binding and Activation Has No Effect on \\ Candida Growth and Viability}

Further experiments aimed to study the effect of platelet binding and subsequent activation on fungal growth and viability. For that purpose, the different Candida species were incubated for $18 \mathrm{~h}$ in RPMI medium with increasing numbers of platelets. Fungal growth was measured by quantification of biomass by calcofluor staining; incubation with the corresponding amount of PPP was used as control.

No growth reduction was visible, even with a 10,000fold excess of platelets (Fig. 5a). In some samples, fungal growth was even higher than in the controls, presumably due to the higher nutrient content provided by the platelet preparation.

To confirm these results, the fungal cells were stained with propidium iodide, which does not diffuse into living cells, but only stains dead cells. No increased propidium iodide staining of the fungal cells was visible after incubation with the platelets (Fig. 5b).
The inability of platelets to affect fungal viability and growth could be due to the limited platelet activation, which in turn would lead to limited release of plateletderived antimicrobial substances. To determine whether these antimicrobial substances have the potential to damage Candida, we used lysed platelets where all antimicrobial granule content was set free. However, the platelet lysate was also not able to reduce fungal biomass or increase fungal damage compared to the PPP control (Fig. 5c, d).

\section{Discussion}

Platelets were recently identified to contribute to immune response against invading pathogens, which may lead to multifaceted antimicrobial effects [27], but also to excessive inflammation and thrombosis [28]. To investigate the interaction of platelets with Candida spp. and putative consequences thereof might gain new insights into the pathogenesis of invasive Candida infections. For that reason, the present study focused on clinical Candida isolates of pathogenic species, and on the investigation of different morphotypes (yeast cells, hyphae, biofilm) as well as secreted factors.

Platelets moderately adhered to yeast cells of all tested Candida species. C. auris and C. glabrata were comparatively high platelet binders, whereas platelets rarely attached to C. tropicalis and C. rugosa. These results are consistent with the results of previous reports: Maisch and Calderone reported that Candida spp. were able to adhere to aggegated platelets, and mentioned the highest binding rate for C. albicans (C. glabrata was not tested), while C. tropicalis and C. krusei displayed a much lower adherence [29, 30]. In contrast, Robert et al. [11] demonstrated rapid adherence of all tested Candida species (albicans, glabrata, tropicalis, parapsilosis) to platelets in a murine model, with only marginal differences between the strains. A difference between human and murine platelets might be a reason for this discrepancy.

Platelets were also able to bind to Candida (pseudo-) hyphae, with no striking differences between the tested Candida species. In biofilms, the fungus is surrounded by an extracellular matrix that decreases penetration of some host immune factors and reduced phagocytic activity against Candida [31, 32]. However, in our experiments, platelets attached to biofilms of all tested species.

Regardless of the fungal growth morphology, nearly all Candida-bound platelets were activated as confirmed 


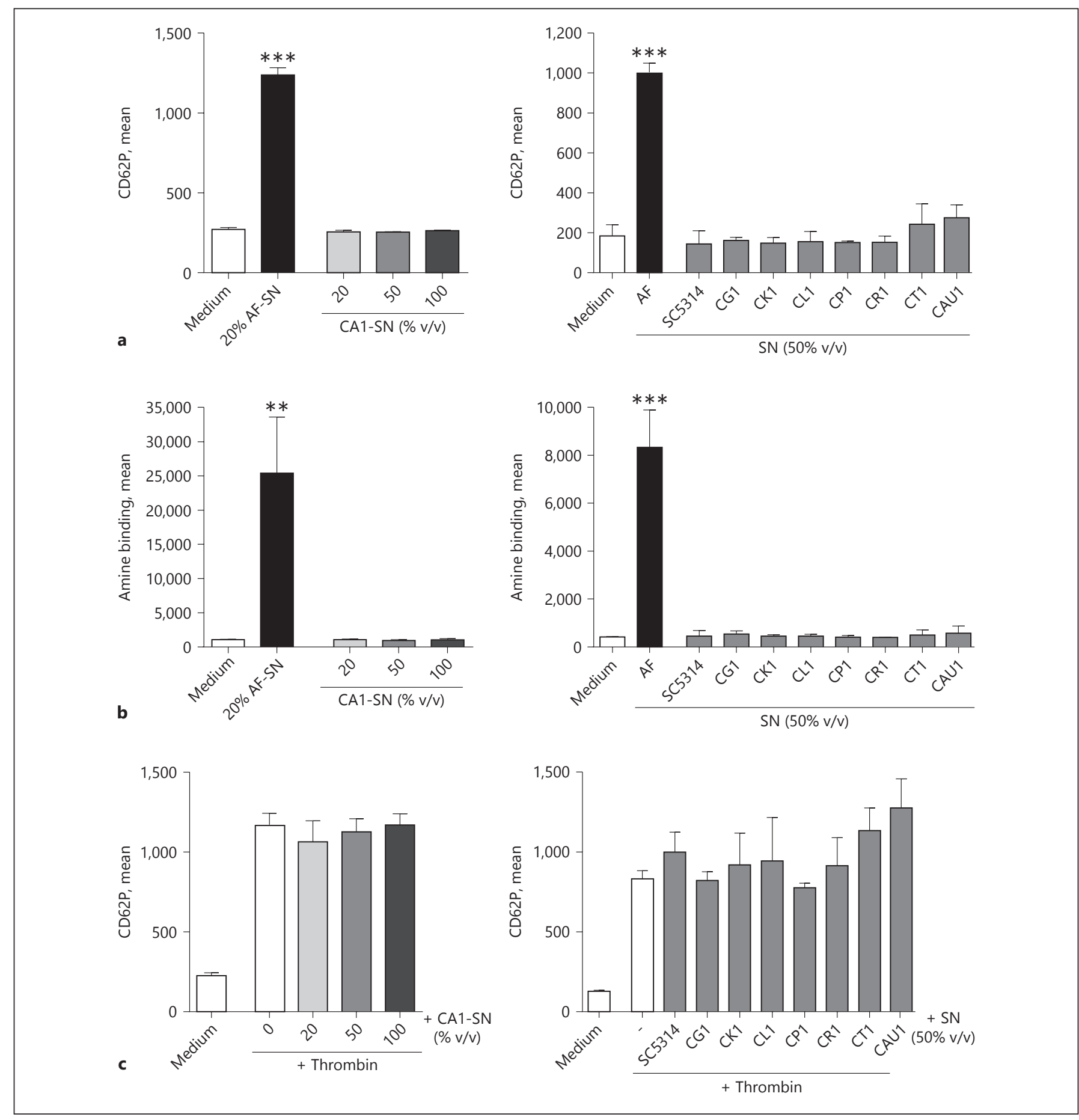

Fig. 4. Influence of Candida culture supernatants on platelet activity and viability. a, b Human platelets were incubated with medium, thrombin, or culture supernatants (SN) from Aspergillus fumigatus (AF), Candida albicans (CA1, SC5314), glabrata (CG1), krusei (CK1), lusitaniae (CL1), parapsilosis (CP1), rugosa (CR1) tropicalis (CT1) or auris (CAU1) for $90 \mathrm{~min}$. Surface exposure of $\mathrm{CD} 62 \mathrm{P}$ as a marker for platelet activity (a) and amine binding as marker for platelet viability (b) were assessed by flow cytometry. c A putative inhibitory influence of Candida supernatants on throm- bin-induced platelet activation was studied by incubating human platelets with both thrombin and culture supernatants from Candida spp. The CD62P signal was quantified by FACS. Each experiment was performed in triplicates and repeated at least 3 times with different donors; representative results are shown here. Activity of samples with Candida were compared to medium control by one-way analysis of variance; * $p<0.05$; $^{* *} p<0.01$; ${ }^{* * *} p<0.005$; $* * * * p<0.001$. 
Fig. 5. Effects of platelets on proliferation and viability of Candida. C. albicans (CA1), C. glabrata (CG1), C. parapsilosis (CP1), C. tropicalis (CT1) or C. lusitaniae (CL1) were incubated with increasing numbers of platelets $(\mathbf{a}, \mathbf{b})$ or lysed platelets $(\mathbf{c}, \mathbf{d})$ for 18 h. Fungal biomass $(\mathbf{a}, \mathbf{c})$ and fungal damage (b, d) were assessed by calcofluor or propidium iodide staining, respectively; parallel samples incubated with the same amount of platelet-poor plasma were set as $100 \%$. Ethanol (EtOH)-treated Candida served as positive control for the fungal damage assays. Each experiment was performed in triplicate; representative results are shown here. ${ }^{*} p<0.05,{ }^{* *} p<0.01,{ }^{* * *} p<0.001$.

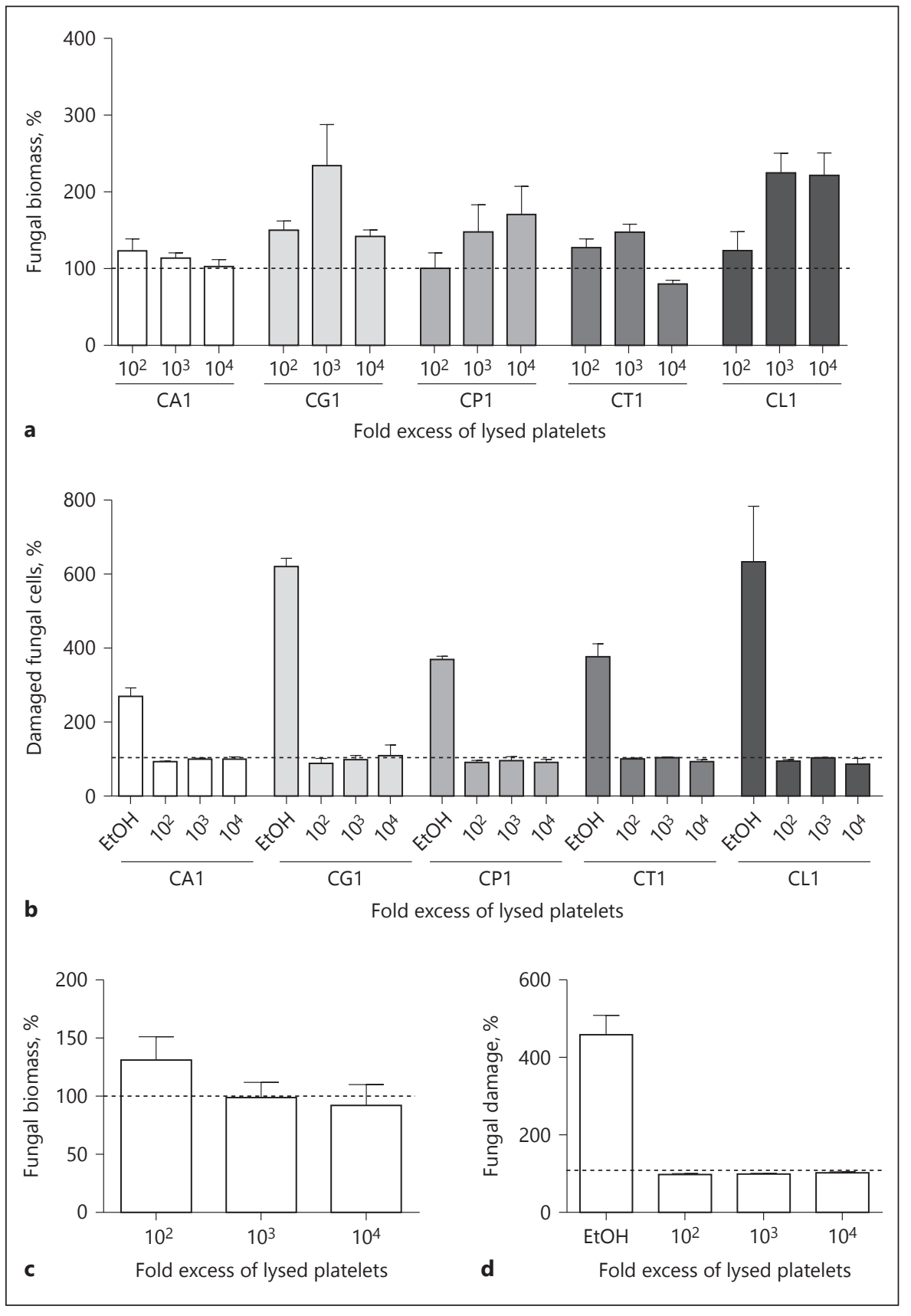

by shape change and CD62P surface expression; nonadhered platelets were not activated. The issue of Candida-induced platelet activation is controversially discussed in literature $[19,24,33-36]$. Similar to our own results, Robert et al. [11,36] described that attachment of platelets to Candida spp. yeast cells and germ tubes resulted in activation-dependent morphological changes. Other studies confirmed the inability of C. albicans to aggregate platelets $[33,35]$. However, Willcox et al.
[19] reported that, with the exception of C. albicans, all other tested species (C. tropicalis, C. parapsilosis, C. krusei) were able to induce platelet aggregation. Skerl et al. [34] described that pre-incubation with plasma (presumably as a source of complement factors for opsonisation) is necessary before cell wall components of C. albicans or C. krusei induce platelet aggregation. These results could not be confirmed by Klotz et al. [33] who reported that cell wall fragments of $C$. albicans did not 
induce aggregation even after pre-incubation with plasma. Other investigators reported that the presence of $C$. albicans even repressed the thrombin-, ADP- or collagen-induced platelet activation [35], which could not be confirmed by our studies.

According to our results, different experimental settings explain the divergence of results. We show that analyzing the complete platelet population versus the subset of Candida-bound platelets led to opposing results. Other variants to explain contrasting findings in the literature might be the used Candida isolate(s). Since a lack of platelet activation might be advantageous for the fungus in IC, clinical isolates might be hypothesized to lead to different results than laboratory strains. However, we did not find significant differences between the clinical isolates that we tested here and the 2 laboratory strains Sc5314 and SN152.

Complement is another factor that might contribute to these divergent findings. This ancient and complex system contributes substantially to a multitude of processes in the immune network and in cellular homeostasis. It is involved in defense against bacteria, viruses, fungi and parasites but can also cause severe tissue damage by excessive inflammatory reactions. Complement closely interacts with diverse immune cells including platelets, thereby bridging innate and acquired immunity $[37,38]$.

As platelets can intensively crosstalk with other immune cells, the platelet response to contact with C. albicans was also investigated in a whole-blood model, which mirrors the in vivo situation more closely than PRP. According to our results, the binding rate to Candida was the same as for isolated platelets, and activation was again confined to the subset of platelets with direct contact to the Candida surface, suggesting that presence of other blood cells does not modify the platelet response to C. albicans.

Not only the fungal surface but also secreted factors might modulate platelet activity. Although Candida secretes a variety of soluble factors, Candida culture supernatants could neither induce platelet activation nor repress thrombin-induced platelet stimulation in our experiments. Other reports confirm that the culture supernatants of $C$. albicans do not lead to the inhibition of aggregation [35]. This inactivity of secreted compounds toward platelets is in striking contrast to our former results with A. fumigatus culture supernatants, which clearly activated platelets in a dose-dependent manner [17]. Diversity in quantity and identity of the generated metabolites between the fungal species might explain this difference. Again, the lack of platelet activation by Candida-derived soluble factors could partly explain fungal survival in blood and the frequency of Candida sepsis.

Activated platelets release various antimicrobial peptides termed PMPs (platelet microbicidal proteins) including thrombocidins and kinocidins, which are chemokines with direct microbicidal activity. These effector molecules show in vitro activity against diverse bacteria and fungi including Cryptococcus neoformans and Candida spp. [39, 40]. However, despite all known antimicrobial activities of stimulated platelets, our experiments revealed neither suppression of fungal proliferation nor fungal killing. This cannot be fully explained by the low frequency of activated platelets, as it was also observed with platelet lysates where all antimicrobial granule content was set free. Contrary to our results, antifungal activity of PMPs against Candida has been reported in several studies $[19,39,41,42]$. Yeaman et al. [42] described differences in susceptibility between different Candida species. C. albicans and C. tropicalis were the most sensitive to PMPs, followed by C. glabrata, whereas C. parapsilosis was resistant to PMP-induced killing. It was suggested that PMPs contribute to host antifungal defense, since PMP-resistant C. albicans strains caused more severe endocarditis in rabbits than PMP-sensitive strains [22]. In contrast, Drago et al. [41] reported that the supernatant of clotted PRP was capable to inhibit growth of C. albicans, but fungal viability was not affected. Further experiments are necessary to clarify the picture and to evaluate Candida species strain specificity.

Taken together, our results suggest that the role of platelets in Candida infections must be considered nuanced. The comparatively low capacity of platelets to interact with Candida and to attack the invader might crucially affect the outcome of disease. Thus, a platelet-targeted therapy might represent an interesting approach for a supportive immunotherapy of IC.

\section{Disclosure Statement}

The authors declare that they do not have any potential conflicts of interest to disclose.

\section{Funding Source}

This work was supported by the "Oesterreichische Nationalbank” (Jubiläumsfonds Project Nr. 15352). 


\section{References}

1 Lamoth F, Lockhart SR, Berkow EL, Calandra $\mathrm{T}$ : Changes in the epidemiological landscape of invasive candidiasis. J Antimicrob Chemother 2018;73:i4-i13.

2 Rambach G, Speth C, Lass-Flörl C: Changing epidemiology of invasive fungal infections in critically ill patients in the intensive care unit. J Invasive Fungal Infect 2010;3:116-122.

-3 Bassetti M, Righi E, Montravers P, Cornely OA: What has changed in the treatment of invasive candidiasis? A look at the past 10 years and ahead. J Antimicrob Chemother 2018; 73:i14-i25.

-4 Rambach G, Oberhauser H, Speth C, LassFlörl C: Susceptibility of Candida species and various moulds to antimycotic drugs: use of epidemiological cutoff values according to EUCAST and CLSI in an 8-year survey. Med Mycol 2011;49:856-863.

$>5$ Lamoth F, Kontoyiannis DP: The Candida auris alert: facts and perspectives. J Infect Dis 2018;217:516-520.

6 Kehrel B, Jurk K: Platelets at the interface between hemostasis and innate immunity. Transfus Med Hemother 2004;31:379386.

7 Speth C, Loffler J, Krappmann S, Lass-Flörl C, Rambach G: Platelets as immune cells in infectious diseases. Future Microbiol 2013;8: 1431-1451.

$>8$ Semple JW, Italiano JE Jr, Freedman J: Platelets and the immune continuum. Nat Rev Immunol 2011;11:264-274.

$>9$ Nurden AT: Platelets, inflammation and tissue regeneration. Thromb Haemost 2011; 105(suppl 1):S13-S33.

10 Christin L, Wysong DR, Meshulam T, Hastey R, Simons ER, Diamond RD: Human platelets damage Aspergillus fumigatus hyphae and may supplement killing by neutrophils. Infect Immun 1998;66:1181-1189.

11 Robert R, Nail S, Marot-Leblond A, Cottin J, Miegeville M, Quenouillere S, Mahaza C, Senet JM: Adherence of platelets to Candida species in vivo. Infection and Immunity 2000; 68:570-576.

$>12$ Chang FY, Singh N, Gayowski T, Wagener MM, Mietzner SM, Stout JE, Marino IR: Thrombocytopenia in liver transplant recipients: predictors, impact on fungal infections, and role of endogenous thrombopoietin. Transplantation 2000;69:70-75.

$\checkmark 13$ Rosenhagen M, Feldhues R, Schmidt J, Hoppe-Tichy T, Geiss HK: A risk profile for invasive aspergillosis in liver transplant recipients. Infection 2009;37:313-319.

-14 de Araujo Motta F, Dalla-Costa LM, Dominguez Muro M, Lenzi A, Picharski GL, Burger M: Risk adjustment for congenital heart surgery score as a risk factor for Candidemia in children undergoing congenital heart defect surgery. Pediatr Infect Dis J 2016;35:1194-1198.
15 Perkhofer S, Kehrel BE, Dierich MP, Donnelly JP, Nussbaumer W, Hofmann J, von EC, LassFlörl C: Human platelets attenuate Aspergillus species via granule-dependent mechanisms. J Infect Dis 2008;198:1243-1246.

16 Perkhofer S, Kainzner B, Kehrel BE, Dierich MP, Nussbaumer W, Lass-Flörl C: Potential antifungal effects of human platelets against zygomycetes in vitro. J Infect Dis 2009;200: 1176-1179.

17 Speth C, Hagleitner M, Ott HW, Würzner R, Lass-Flörl C, Rambach G: Aspergillus fumigatus activates thrombocytes by secretion of soluble compounds. J Infect Dis 2013;207: 823-833.

18 Lass-Flörl C, Wiedauer B, Mayr A, Kirchmair M, Jenewein I, Ledochowski M, Dierich MP: Antifungal properties of 5-HT (serotonin) against Aspergillus spp. in vitro. Int J Med Microbiol 2005;26:335-337.

19 Willcox M, Webb B, thakur A, Harty D: Interactions between Candida species and platelets. J Med Microbiol 1998;47:103-110.

20 Yeaman M: The role of platelets in antimicrobial host defense. Clin Infect Dis 1997;25: 951-970.

21 Woth G, Tokes-Fuzesi M, Magyarlaki T, Kovacs GL, Vermes I, Muhl D: Activated platelet-derived microparticle numbers are elevated in patients with severe fungal (Candida albicans) sepsis. Ann Clin Biochem 2012;49: 554-560.

22 Yeaman MR, Soldan SS, Ghannoum MA, Edwards JE Jr, Filler SG, Bayer AS: Resistance to platelet microbicidal protein results in increased severity of experimental Candida albicans endocarditis. Infect Immun 1996;64: 1379-1384.

23 Yeaman MR, Cheng D, Desai B, Kupferwasser LI, Xiong YQ, Gank KD, Edwards JE Jr, Bayer AS: Susceptibility to thrombin-induced platelet microbicidal protein is associated with increased fluconazole efficacy against experimental endocarditis due to Candida albicans. Antimicrob Agents Chemother 2004; 48:3051-3056.

24 Bertling A, Niemann S, Uekotter A, Fegeler W, Lass-Flörl C, von EC, Kehrel BE: Candida albicans and its metabolite gliotoxin inhibit platelet function via interaction with thiols. Thromb Haemost 2010;104.

25 Noble SM, Johnson AD: Strains and strategies for large-scale gene deletion studies of the diploid human fungal pathogen Candida albicans. Eukaryot Cell 2005;4:298-309.

26 Odds FC, Brown AJ, Gow NA: Candida albicans genome sequence: a platform for genomics in the absence of genetics. Genome Biology 2004;5:230.

27 Yeaman MR: Platelets: at the nexus of antimicrobial defence. Nat Rev Microbiol 2014;12: 426-437.

$>28$ Wagner DD, Burger PC: Platelets in inflammation and thrombosis. Arterioscler Thromb Vasc Biol 2003;23:2131-2137.
29 Maisch PA, Calderone RA: Adherence of Candida albicans to a fibrin-platelet matrix formed in vitro. Infect Immun 1980;27:650656.

\$3 Maisch PA, Calderone RA: Role of surface mannan in the adherence of Candida albicans to fibrin-platelet clots formed in vitro. Infect Immun 1981;32:92-97.

31 Chandra J, McCormick TS, Imamura Y, Mukherjee PK, Ghannoum MA: Interaction of Candida albicans with adherent human peripheral blood mononuclear cells increases $C$. albicans biofilm formation and results in differential expression of pro- and anti-inflammatory cytokines. Infect Immun 2007;75: 2612-2620.

32 Katragkou A, Kruhlak MJ, Simitsopoulou M, Chatzimoschou A, Taparkou A, Cotten CJ, Paliogianni F, Diza-Mataftsi E, Tsantali C, Walsh TJ, Roilides E: Interactions between human phagocytes and Candida albicans biofilms alone and in combination with antifungal agents. J Infect Dis 2010;201:1941-1949.

33 Klotz SA, Harrison JL, Misra RP: Aggregated platelets enhance adherence of Candida yeasts to endothelium. J Infect Dis 1989;160: 669-677.

34 Skerl KG, Calderone RA, Sreevalsan T: Platelet interactions with Candida albicans. Infect Immun 1981;34:938-943.

35 Carvalho Neiva TJ, dos Santos JI: Effect of pathogenic yeasts on human platelet aggregation. Braz J Infect Dis 2003;7:370-374.

36 Robert R, Mahaza C, Miegeville M, Ponton J, Marot-Leblond A, Senet JM: Binding of resting platelets to Candida albicans germ tubes. Infection and Immunity 1996;64:3752-3757.

37 Ricklin D, Hajishengallis G, Yang K, Lambris JD: Complement: a key system for immune surveillance and homeostasis. Nature immunology 2010;11:785-797.

38 Speth C, Rambach G, Wurzner R, Lass-Florl C, Kozarcanin H, Hamad OA, Nilsson B, Ekdahl KN: Complement and platelets: mutual interference in the immune network. Mol Immunol 2015;67:108-118.

39 Tang Y, Yeaman M, Selsted ME: Antimicrobial peptides from human platelets. Infection and Immunity 2002;70:6524-6533.

-40 Yeaman MR: Platelets in defense against bacterial pathogens. Cell Mol Life Sci 2010;67: 525-544.

41 Drago L, Bortolin M, Vassena C, Taschieri S, Del FM: Antimicrobial activity of pure platelet-rich plasma against microorganisms isolated from oral cavity. BMC Microbiol 2013; 13:47.

42 Yeaman MR, Ibrahim AS, Edwards JE Jr, Bayer AS, Ghannoum MA: Thrombin-induced rabbit platelet microbicidal protein is fungicidal in vitro. Antimicrob Agents Chemother 1993;37:546-553. 